\title{
Docking and 3D-QSAR Studies on Some HCV NS5b Inhibitors
}

\author{
Yasmine Shafike Moemen ${ }^{1,}$, , Ahmed Mahmoud El-Nahas ${ }^{2}$, Ahmed Helmy Ebraheem Hassan ${ }^{3}$, \\ Safwat Abdel-Azeim ${ }^{4}$, Serry Atta Atta El-Bialy ${ }^{5}$ \\ ${ }^{1}$ Clinical Pathology Department, National Liver Institute, Menoufia University, Shebin El-Kom, Egypt \\ ${ }^{2}$ Chemistry Department, Faculty of Science, Menoufia University, Shebin El-Kom, Egypt \\ ${ }^{3}$ Medicinal Chemistry, Faculty of Pharmacy, Mansoura University, Mansoura, Egypt \\ ${ }^{4}$ Modelling Laboratory, Division of Physical Science \& Engineering, Jeddah, SA \\ ${ }^{5}$ Pharmaceutical Organic Chemistry, Faculty of Pharmacy, Mansoura University, Mansoura, Egypt
}

Email address:

yasmine_moemen@liver-eg.org (Y.S. Moemen)

${ }^{*}$ Corresponding author

\section{To cite this article:}

Yasmine Shafike Moemen, Ahmed Mahmoud El-Nahas, Ahmed Helmy Ebraheem Hassan, Safwat Abdel-Azeim, Serry Atta Atta El-Bialy. Docking and 3D-QSAR Studies on Some HCV NS5b Inhibitors. Journal of Drug Design and Medicinal Chemistry.

Vol. 3, No. 4, 2017, pp. 49-59. doi: 10.11648/j.jddmc.20170304.11

Received: September 22, 2016; Accepted: August 7, 2017; Published: October 23, 2017

\begin{abstract}
A theoretical study has been carried out to interpret and support experimental findings regarding inhibition mechanism of HCV NS5b. Twenty-five HCV NS5b inhibitors were docked by QM-Polarized Ligand Docking (QPLD) technique. The comparative molecular field analysis (CoMFA) and comparative molecular similarity indices analysis (CoMSIA) methods were used to derive 3D-QSAR models for the selected inhibitors. The CoMFA and CoMSIA models show good cross-validated $\left(\mathrm{Q}^{2}\right)$ and non-cross-validated $\left(\mathrm{R}^{2}\right)$ coefficients for the suggested inhibitors of $0.43,0.98$ and $0.65,0.99$, respectively. The inhibition mechanism was explored and validated. Details of the interactions between the inhibitors and HCV NS5b are given in terms of steric, electrostatic, hydrophobic, hydrogen bonding fields. Enhancing potency via substitutions at positions, which were explored based on these parameters. A good correlation was found between 3D-QSAR and docking results.
\end{abstract}

Keywords: QPLD, Docking, 3D-QSAR, CoMFA, CoMSIA, HCV NS5b Inhibitors

\section{Introduction}

Hepatitis C Virus (HCV) is a genus of the Flaviviridae family with six major genotypes. It includes a large number of subtypes within each genotype [1]. Worldwide distribution of HCV genotypes includes genotype 1 (Japan, Europe and North America), genotype 2 (Japan and North America), genotype 3 (Indian subcontinent), genotype 4 (North Africa and the Middle East), genotype 5 (South Africa) and genotype 6 (South East Asia) [2, 3].

$\mathrm{HCV}$ epidemiology is approximately $3 \%$ prevalent worldwide [4, 5], one-fifth of the globally HCV carriers live in the Middle East [2]. HCV infection was estimated to be about $20 \%$ of Egyptians with a newly half million infections per year [6-8]. Egypt has the highest globally incidence of
$\mathrm{HCV}$ infection [9-13] and it varies from 9 to $50 \%$ in certain rural areas due to the specific modes of infection [5]. While the mechanism of infection is fully acquainted, the mechanism of viral entry and replication are not completely understood [8]. HCV consists of different enzymes, though there are many clues that HCV polymerase is the maestro enzyme in viral replication process $[14,15]$.

Similar to any DNA polymerases, HCV NS5b adopts the architecture of a right hand with "thumb," "palm," and "fingers" domains. "The palm domain catalyzes the phosphoryl transfer reaction, whereas the fingers domain participates in interactions with the incoming nucleoside triphosphate as well as the template base to which it is paired [14]. However, the thumb domain plays a role in positioning the duplex DNA and in processivity and translocation [14]. $\mathrm{HCV}$ NS5b is an interesting target for antiviral therapy with 
limited side effects. It was the subject of extensive trails to design both nucleoside and non-nucleoside inhibitors $[15,16]$. Different studies discussed various potential sites of HCV NS5b: Thumb pocket-I (Pro495, Pro496, Val499), Thumb pocket-II (Leu419, Met423), Palm pocket-I (Asn411, Met414, Tyr448) and the active site (Ser282) [17-24].

Molecular docking represents the basics of rational drug design [25], since it predicts the appropriate structure conformation for the potential target. The first docking study was done by Levinthal et al. [26] to predict the conformations of hemoglobin fibers.

Quantitative Structure Activity Relationship (QSAR) was found to be a good tool for reproducing activity of various inhibitors toward particular receptors [27]. The threedimensional QSAR (3D-QSAR) is a good representative of inhibitor-receptor interactions with their bioactive conformations [28, 29].

The current work aims at studying some HCV NS5b inhibitors and their interaction with four potential sites (Thumb pocket-I, -II, Palm pocket-I and the active site) using various docking protocols and constructing 3D-QSAR models, such compounds were selected for the present work because of their potency, diversity and selectivity besides the high resolution of the experimental complex structure (2HAI). This investigation helps in understanding of inhibition efficiency of the selected compounds and tailoring of new inhibitors.

\section{Methodology}

A set of twenty five inhibitors of diverse activities was selected from a previous experiment [18]. These inhibitors are displayed in Figure 1. They were selected based on their structure diversity, hydrogen bonding ability, hydrophobic moieties, aromatic rings, substitution patterns and their potency toward HCV NS5b allosteric pockets. All inhibitors were sketched by Epike v. 2 module [30]. Then, stereoisomerism and tautomerization options were selected with OPLS- 2005 force field. A set of 88 conformers were produced with Ligprep module embedded in Schrodinger suite. These ligands were docked by QPLD method.

QPLD includes Glide and QSite packages. The QPLD algorithm launches with Glide docking job which produces several protein-ligand complexes. After that, QSite performs a single-point energy calculation on each complex treating the ligand with ab initio methods and deriving partial atomic charges using electrostatic potential fitting as QM/MM environment. Glide then re-docks the ligand using each of the ligand charge sets calculated by QSite. Finally QPLD algorithm gives the most energetically favorable pose. The fully automated algorithm is calibrated to provide useful settings according to the user's need [31, 32].

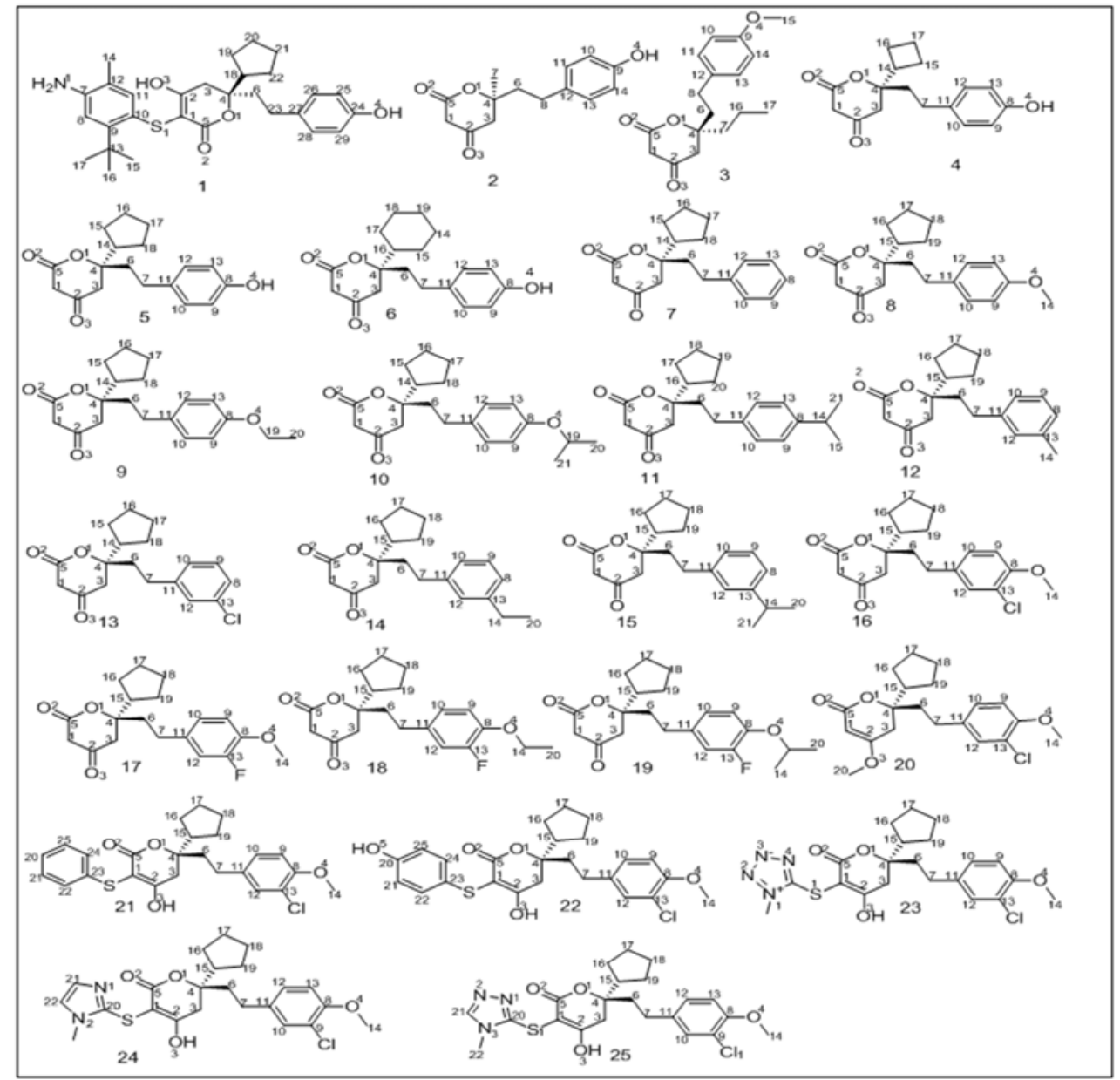

Figure 1. Chemical structures of the investigated compounds. 
The mixed QM/MM approach in QPLD is more accurate especially in the case of metalloproteins [31]. The accuracy in determining binding modes from Glide is about 82\% [33] which makes it an appropriate tool for the current docking study. Glide standard error was found to be $\sim 2 \mathrm{kcal} / \mathrm{mol}$.

Before docking, all missing atoms were adjusted to match the original protein structure. Protein preparation wizard was used to assign all bond orders, hydrogen atoms, formal charges on the metallic centers. The neighboring atoms were also assigned and all water molecules were deleted. Prediction of ionization and tautomeric states of the metal groups at $\mathrm{pH} 7$ was performed. A minimization with $0.1 \AA$ RMSD and OPLS-2005 force field was applied. Finally, the whole complex, 2HAI, was split into protein and inhibitor. All inhibitors were docked using Glide XP score in QPLD [34]. Docking validation can be done by calculating coefficient correlation between $\mathrm{IC}_{50}[35,36]$ or $-\log \mathrm{IC}_{50}$ $\left(\mathrm{pIC}_{50}\right)$ [37-39] and docking scores.

All conformations were analyzed using LPC package [40] to determine the best interaction. The best docked conformations for all inhibitors docked by QPLD were also used for Autodock (Genetic algorithm) and Glide XP calculations. Unfortunately, the scores derived from both methods showed no good correlation with the experiment, see supporting material. Molegro molecular view [33] and Chimera [41] were used to extract the docked inhibitors from their receptors and for their graphic representation, respectively.

The 3D-QSAR models were performed for the most active conformations extracted from docking. These 3D-QSAR models were derived from comparative molecular field analysis (CoMFA) and comparative molecular similarity indices analysis (CoMSIA) methodologies with PLS algorithm[42]. The 3D-QSAR calculations were performed using Sybyl v. $\mathrm{x} 2$ which is based on the Tripos force field with a distance-dependent dielectric, the Powell conjugate gradient algorithm and Gasteiger-Hückel charges [43].

CoMFA represents a 3D cubic grid with $2 \AA$ spacing and 4 $\AA$ automatic extension away from the investigated molecules in all three $\operatorname{axes}(\mathrm{X}, \mathrm{Y}, \mathrm{Z}$ directions). Lennard-Jones and Columbic potentials were calculated to determine the steric and electrostatic interaction fields at each grid intersection. An sp3 carbon atom with a radius of $1.52 \AA$ bearing +1 charge was used as a probe atom to calculate the CoMFA fields [44]. CoMFA contour maps were plotted to analyze steric and electrostatic features to shed light into the strength of binding between the inhibitors and receptor as determined from docking.

CoMSIA calculates five similarity descriptors namely steric, electrostatic, hydrophobic, hydrogen bond donor and acceptor[45]. Gaussian type function was employed to calculate similarity indices at all lattice points. Smoothening function applied by a default value of 0.3 . The probe atom with charge +1 , hydrophobicity +1 , and hydrogen bond donor and acceptor property of +1 was used. The steric indices were related to third power of the radii, partial atomic charges were used to derive the electrostatic fields. Atom based parameters computed hydrophobic descriptors and rule based method was used to get the hydrogen bond donor and acceptor fields [46].

\section{Results and Discussion}

The RNA dependent RNA polymerase (RdRP) adopts a "closed-right hand" conformation with "thumb", "fingers" and "palm" sub-domains as depicted in Figure 2. The thumb domain (Thumb pocket-I) is an allosteric binding site adjacent to the Guanosine-5'-triphosphate (GTP) which is an allosteric regulator of RNA synthesis or the GTP surface binding site may provide an oligomerization surface for NS5b [47]. Thumb pocket-I is located $30 \AA$ away from the active site. This domain contains Val37, Leu392, Ala393, Ala395, Ala396, Thr399, Ile424, Leu425, His428, Phe429, Leu492, Gly493, Val494, Pro495, Trp500 and Arg503 amino acids. The GTP-binding sites is located about 10-15 $\AA$ away from Thumb pocket-II. This pocket includes Leu419, Arg422, Met423, His475, Ser476, Tyr477, Ile482, Val485, Leu497, Leu489, Arg501, Trp528 and Lys533 amino acids. The fingers domain is characterized by two regions: inner and outer. The former consists of a bundle of $\alpha$-helices around the palm sub-domain. However, the outer region stands away from the palm area. In the latter region, the nucleotide binding site exists near the active site. The palm domain consists of a catalytic active site with highly conserved regions in all RdRP $[48,49]$. The palm region includes Palm pocket-I which consists of Phe193, Tyr195, Pro197, Arg200, Leu204, Ser228, Asn291, Leu314, Asn316, Gly317, Asp318, Val321, Leu360, Ile363, Ser365, Cys366, Ser368, Val370, Leu384, Asn411, Met414, Tyr415, Leu446, Ile447, Tyr448, Gly449, Tyr452, Trp550, Phe551, Ser556 residues [50-52].

\subsection{Docking}

It was reported that the inhibition of the allosteric sites reduces the catalytic activity of the HCV NS5b [15]. Therefore, there is an unavoidable conflict in rational design of drugs targeting these sites [53]. Li et al. [18] and Love et al. [53] designed a lead for these sites. As shown in Figure 2, the blue part of 1 tends to bind with Thumb pocket-I, while the purple area binds to Thumb pocket-II. The red region binds to Palm pocket-I. For rationalizing the experimental findings, QPLD was applied to elucidate the interaction of dihydropyrones derivatives with Thumb pocket-I, -II, Palm pocket-I and the active site.

Docking validation was done by redocking of inhibitor 19 with RMSD of $0.9 \AA$ when compared to its crystal structure, 2HAI. This assumes a good docking [54], see Figure 3. QPLD gives a good correlation between the calculated binding energies and $\mathrm{IC}_{50}$ as emerges from $\mathrm{R}^{2}$ values of $0.68 \rightarrow 0.80$ and $0.82 \rightarrow 0.90$ with Thumb pocket-II and Palm pocket-I, respectively. These values are better than that recorded for Thumb pocket-I, Table 1 . This indicates that most compounds tend to bind strongly with Thumb pocket-II, 
Palm pocket-I rather than with Thumb pocket-I. The investigated inhibitors do not show any activity toward the

active site as listed in the supporting information.
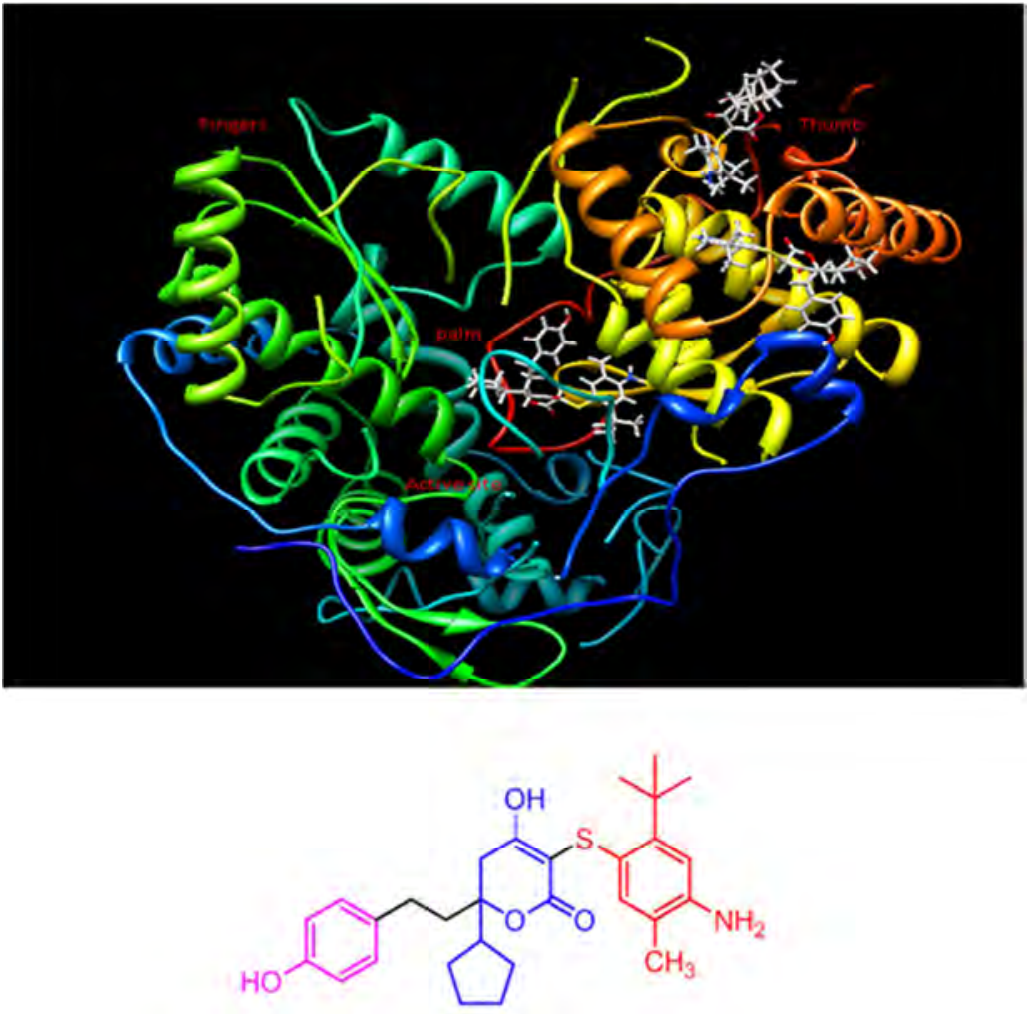

Figure 2. Schematic representation of HCVN5b with allosteric sites and inhibitor 1 bound to Thumb pocket-I, -II, Palm pocket-I. The potent structure 1 is given with different colors to indicate the active parts toward each pocket of HCV NS5b.

The inhibitor 1 was found to interact selectively with Thumb pocket-II which does not exist in the thumb domain of other polymerases (cellular or viral) [55]. The present study agrees with this view where 1 shows a considerable activity toward all allosteric sites rendering it as a lead compound $\left(\mathrm{IC}_{50}=0.93 \mu \mathrm{M}\right)$, Figure 3 . Docking study in the thumb domain, Thumb pocket-I, indicates that the phenol group in compounds 1 and 2 are directed toward the hydrophobic regions (Val485, Leu489 and Val494). Due to the proximity of Thumb pocket-I and -II, they interact similarly with compounds $3-20$ as these interactions will be discussed later. However, the rest of compounds do not show a remarkable activity for Thumb pocket-I, besides compound 25 was excluded from the current docking work because of its tiny $\mathrm{IC}_{50}$ which can't compared with the others, Table 1.

Table 1. Binding affinities (kcal/mol) of 25 inhibitors, calculated by Glide using Quantum polarized docking $(Q P L D)$, and $I C_{50}(\mu M)$ divided into three categories according to $I C_{50}$ values.

\begin{tabular}{lllll}
\hline Inhibitor/R & QPLD_A & QPLD_B & QPLD_C & IC $_{\mathbf{5 0}}$ \\
\hline Category A & & & & \\
2 & -2.83 & -4.55 & -2.13 & 100 \\
3 & -5.92 & -5.63 & -4 & 50 \\
4 & -4.94 & -5.88 & -2.422 & 93 \\
6 & -5.97 & -5.79 & -4.32 & 52 \\
7 & -5.79 & -6.09 & -4.6 & 48 \\
12 & -6.25 & -6.74 & -4.1 & 23 \\
20 & -6.28 & -6.52 & -5.02 & 29 \\
$\mathrm{R}^{2}$ & 0.76 & 0.68 & 0.82 & \\
\hline
\end{tabular}

\begin{tabular}{lllll}
\hline Inhibitor/R & QPLD_A & QPLD_B & QPLD_C & IC $_{\mathbf{5 0}}$ \\
\hline Category B & & & & \\
5 & -4.88 & -6.34 & -3.90 & 8.2 \\
8 & -6.29 & -6.28 & -3.54 & 9.7 \\
9 & -6.85 & -6.67 & -3.43 & 2.9 \\
10 & -6.02 & -6.66 & -1.48 & 1.7 \\
11 & -6.22 & -6.76 & -4.34 & 4.3 \\
13 & -6.18 & -6.48 & -1.50 & 5 \\
14 & -6.19 & -6.84 & -2.94 & 3.6 \\
15 & -5.62 & -6.65 & -3.47 & 1.8 \\
16 & -5.97 & -7.01 & -5.11 & 1 \\
$\mathrm{R}^{2}$ & 0.05 & 0.71 & 0.00 & \\
Category C & & & & \\
1 & -2.12 & -6.58 & -4.2 & 0.93 \\
17 & -6.05 & -6.68 & -4.1 & 0.89 \\
18 & -5.82 & -7.16 & -4.45 & 0.79 \\
19 & -6.01 & -6.89 & -4.97 & 0.53 \\
21 & -1.36 & -7.31 & -5 & 0.56 \\
22 & -4.31 & -8.16 & -5.46 & 0.13 \\
23 & -1.89 & -7.74 & -5.35 & 0.28 \\
24 & -1.35 & -7.58 & -6.2 & 0.14 \\
$\mathrm{R}^{2}$ & 0.14 & 0.80 & 0.90 & \\
\hline
\end{tabular}

For Thumb pocket-II, docking results match the experimental study of Love et al. [55]. As displayed in Figure 3 , the inhibitor 1 when compared to crystal structure, 1OS5, the cyclopentyl group lies in hydrophobic residues (Leu419, Arg422 and Met423), the phenol group is surrounded by Leu497, Ile482. Besides, there are two hydrogen bonds formed by the diketo groups of dihydropyran-2-one with Arg501 and Ser476. Compounds 1-16 react with amino acids 
residues in a similar manner except $2,3,6$, and 12 which

show little differences, see Figure 3.

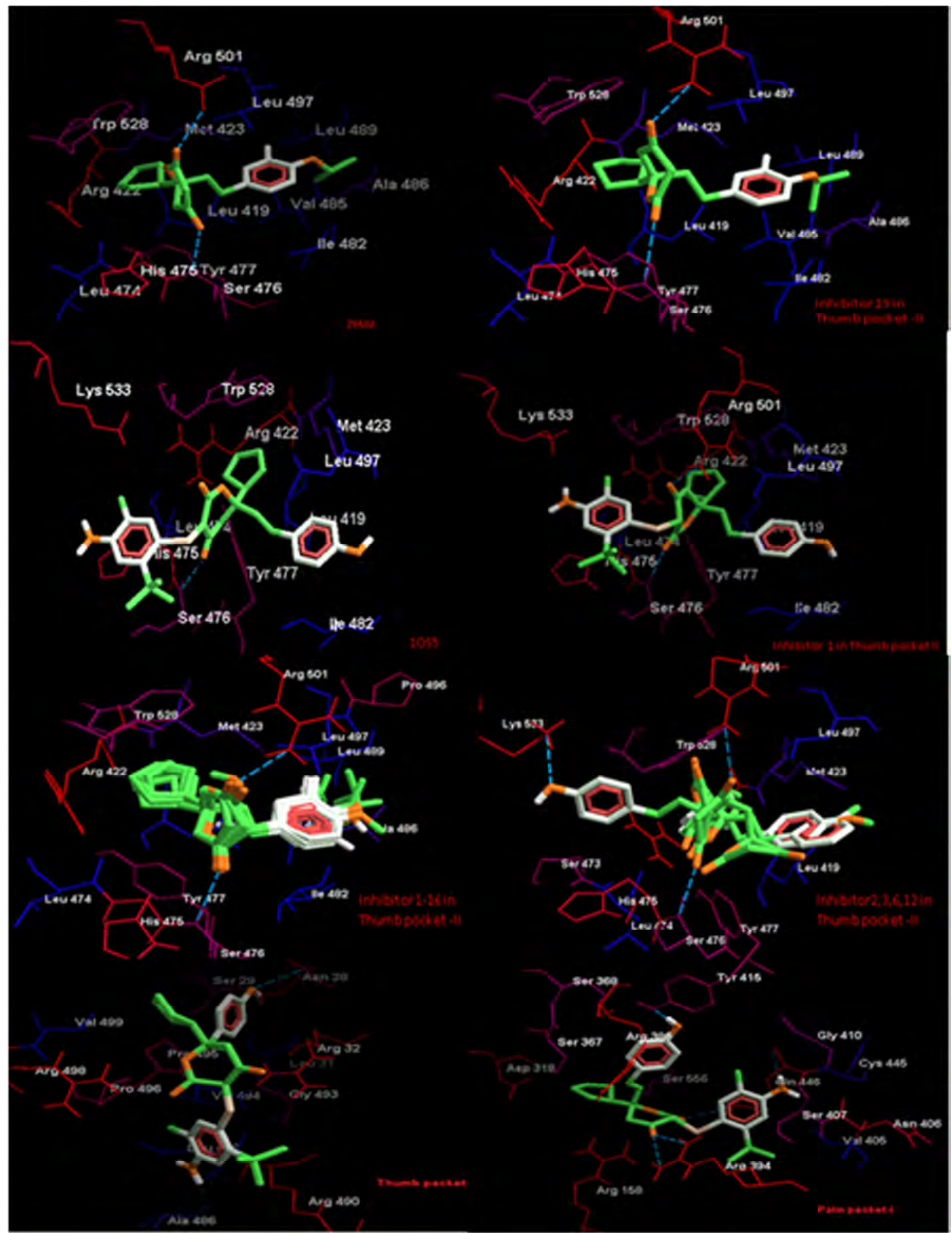

Figure 3. Comparison of different docked structures.

Figure 3. exhibit Crystal structure of (2HAI) compared to docked structure of inhibitor 19, crystal structure (1OS5) compared to docked structure of inhibitor 1 . Thumb pocket-I, Palm pocket-I poses for inhibitor 1, 1-16 except for 2, 3, 6, 12 and 2, 3, 6, 12 also displayed next to it respectively. Amino acid residues appear as thin sticks while ligand atoms are represented as bold sticks. The hydrophobic parts appear in green while the hydrophilic moieties in orange and gray colors. Hydrophilic residues have a red color, while hydrophobic residues have blue color. Atoms of residues are colored according to the hydropathy index proposed by Kyle and Doolittle in 1982 (see http://en.wikipedia.org/wiki/Hydropathy_index for details). The blue dashed line represents the hydrogen bond. 
The phenol group of compound 2 is oriented toward Arg422 and surrounded by Tyr477 and Leu419, parallel stacked with Trp528, hydrogen bonded with Ser476 and Arg422 through $\mathrm{O} 2$ and $\mathrm{O} 4$, respectively. This is confirmed by unfavorable hydrogen bond acceptors (deep red polygon for O2) steric and hydrophobic (yellow and gray for the phenol group) unfavored region, Figure 4. However, compound 2 doesn't fit well with this pocket because of its small size. Therefore, potency of this compound is decreased by 100 times compared to 1 . For compound 3 , the anisole group is located close to the far side of Thumb pocket-II, Val485, Leu489, while there are two hydrogen bonds formed through $\mathrm{O} 3$ and $\mathrm{O} 2$ atoms of the diketo groups of dihydropyran-2-one with Ser476 and Arg501. This inversion of the structure might explain the double potency of 3 compared to $2(51$ vs. $100 \mu \mathrm{M})$. For compound 6 , three hydrogen bonds are formed between Ser476, Arg501, Lys533 and $\mathrm{O} 2, \mathrm{O} 3$ and $\mathrm{O} 4$, respectively, which is explained by unfavorable hydrogen bond acceptors (deep red polygon for O2, O3) and disfavored hydrogen bond acceptor (magenta contour for O4), see Figure 4. This slightly increases the potency $\left(\mathrm{IC}_{50}=52 \mu \mathrm{M}\right)$ compared to 3 . The diketo groups of dihydropyran-2-one in 12 form hydrogen bonds with Ser476 and Arg501 through O2 and O3. Moreover, the toluene moiety interacts with the hydrophobic region (favorable/orange and grey/unfavorable) of Leu419, Met423, Ile482, Val485 and Leu489. This decreases the relevant $\mathrm{IC}_{50}$ to $23 \mu \mathrm{M}$.

Cirrhotic patients or patients with decompensated liver are difficult group to cure, those patients with unmet medical needs will deteriorated through 4 years when they treated with the current approved drug [56].

Due to financial issues, we cannot implement any experimental work on the compounds under study, but using the current docking study as a test set to screen the drug database for isosteres [57], which can be used as potential HCVNS5b drugs for cirrhotic patients [56] in another future work.
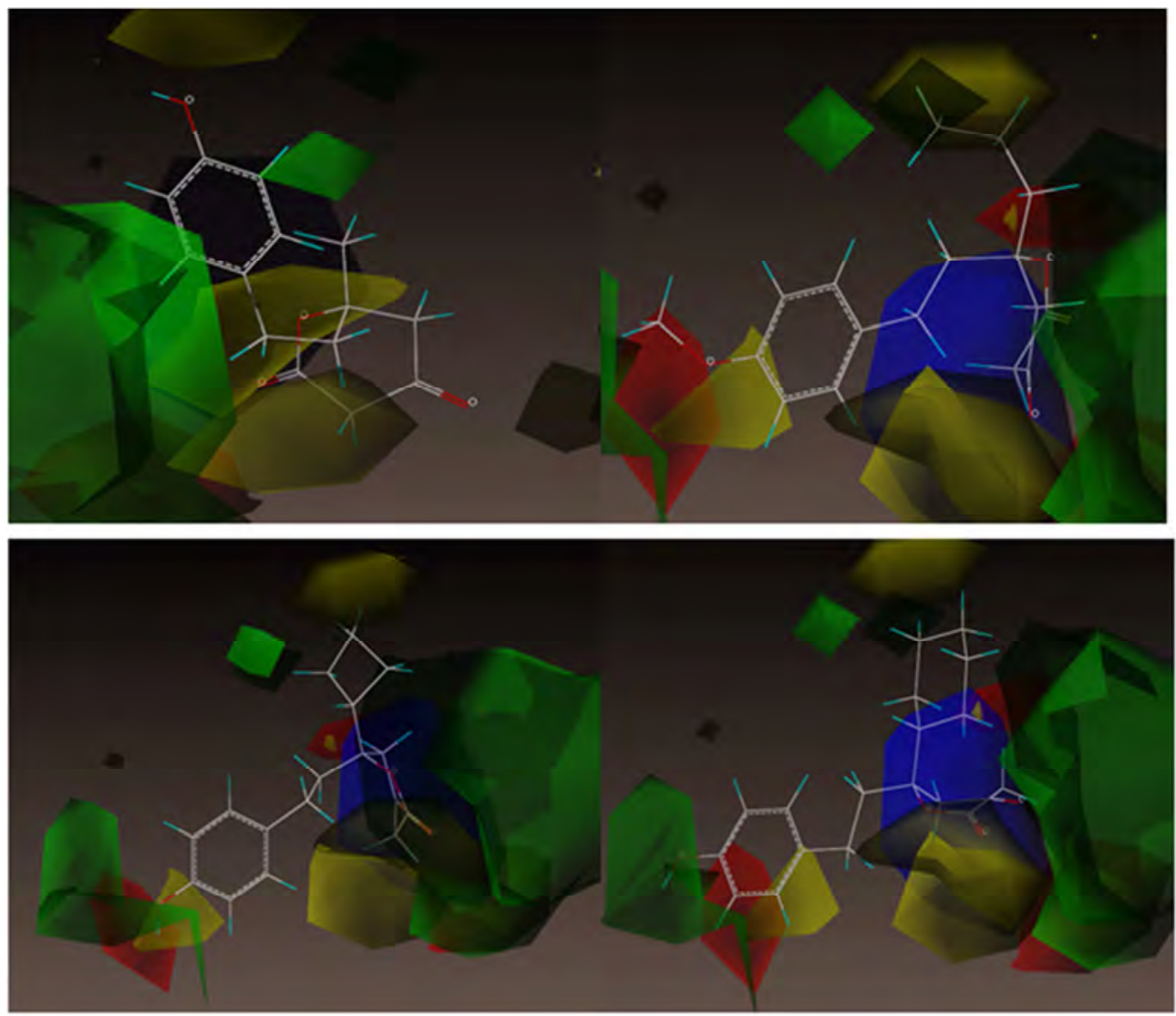

Figure 4. Favorable/unfavorable steric (green/yellow) contours, positive charge favored / negative favored electrostatic (blue, red) contours of COMFA for inhibitors 2,3,4,6 arranged from left to right.

Increasing the size of hydrophobic groups at the para position leads to higher potency (for compounds $6,8 \rightarrow 10$ where $\mathrm{IC}_{50}$ decreases from $52,9.7$ to $1.7 \mu \mathrm{M}$; green, orange polygon sterically favored hydrophobic interaction). Further increase in potency is achieved with addition of a fluorine atom at the meta position of the phenyl ring (for compounds $17-19, \mathrm{IC}_{50}$ decreases from 0.89 to $0.53 \mu \mathrm{M}$; orange favored hydrophobic interaction exist in both meta positions).
The bioactive conformations for Thumb pocket-II were aligned and pharmacophore features were mapped by PharmaGist [58]. Figure 5 displays inhibitor 1 docked in Thumb pocket-I, -II and Palm pocket-I. Hydrophobicity (green), hydrophilicity (orange), aromaticity (red) and nonpolar hydrogen bonds (white) of 1 are shown in Figure 3. The pharmacophore models of bioactive conformations for Thumb pocket-I, -II suggest one aromatic center (phenol 
group as given in pink color in Figure 2), three hydrophobic centers (ethyl group (in black and the chiral carbon of pentane ring (blue)) and two acceptors (two carbonyl groups of dihydropyran-2-one ring (blue)). The aromatic center interacts with the side chains of Tyr 477 for compounds 1 and 2. However, there is no such interaction in other inhibitors. The hydrophobic centers interact with Leu419, Arg422 and Tyr477. Most inhibitors form hydrogen bonds with Ser476, Arg501 residues, except 2, 22 which form hydrogen bonds with other residues. These results coincide with docking data with little differences.

For Palm pocket-I, the pharmacophore model of the bioactive conformations shows three acceptors (two carbonyl groups and oxygen atom of dihydropyran-2-one ring(blue)) and three hydrophobic centers (two ethyl groups (black) and the chiral carbon of pentane ring (blue)). The three acceptors form hydrogen bonds with Leu446, GLY449, while third residue varies from one inhibitor to another. The hydrophobic centers interact with Met414; Leu446 and the third residue might be Tyr415 or Val405. From this pharmacophore models, it is expected to have different bioactive conformations rather than Thumb pocket-I, -II. From docking, most compounds are bent on each other to be accommodated in the Palm pocket-I. This leads to more hydrogen bonds and $\pi-\pi$ stacking than Thumb pocket-II, especially for compounds having more than one aromatic ring such as compounds 1 and 21-25. This explains the activity of such compounds.

\section{Types of interaction per structure and $\mathrm{IC}_{50}$ per compound}

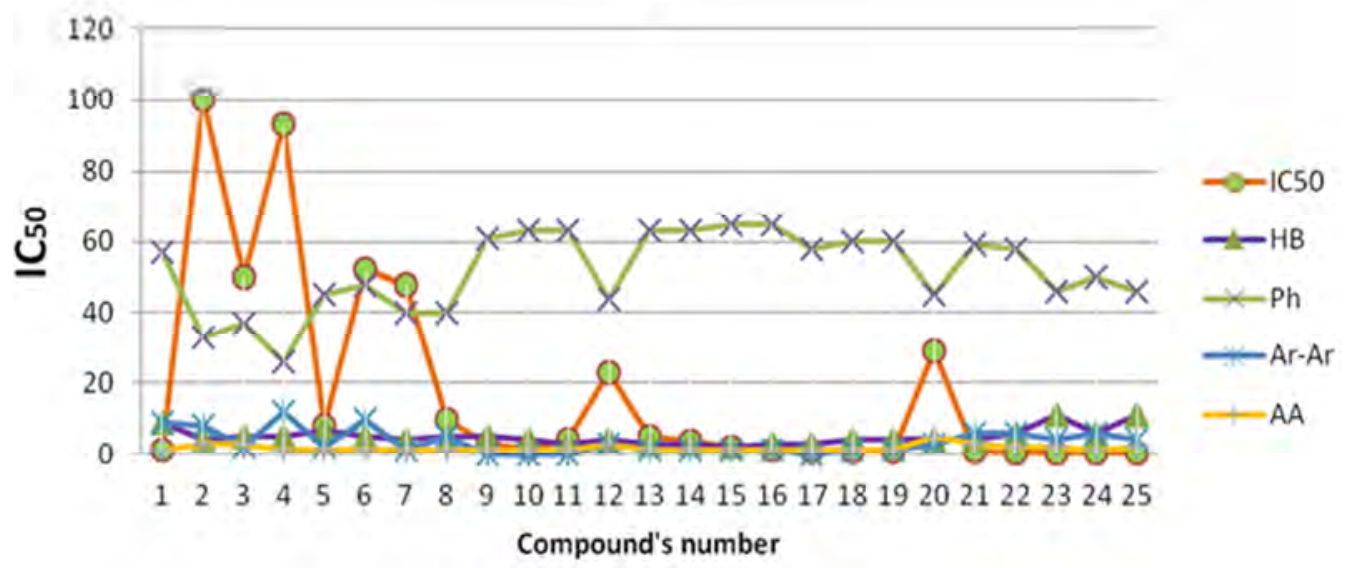

Figure 5. Types of intermolecular interactions: hydrogen bond (HB), hydrophobicity (ph), Aromatic-Aromatic (Ar-Ar), and Acceptor-Acceptor (AA) against $I C_{50}$ of the investigated compounds for Thumb pocket II.

\subsection{D-QSAR}

CoMFA model calculates steric (S) and electrostatic fields (E) with contributions $0.43,0.57$, while CoMSIA model computes steric, electrostatic, hydrogen bond donor (D), hydrogen bond acceptor (A) and hydrophobic (H) with contributions of $0.10,0.26,0.25,0.14$ and 0.24 , respectively, as given in Table 2. The predictive power of the 3-D CoMFA and CoMSIA models were determined from calculating $\mathrm{pIC}_{50}$ of the investigated inhibitors. A maximum difference between the actual and predicted of no more than 0.3 indicates excellent models as listed Table 3 [59].

The contour maps, obtained from CoMFA model, Figure 6, show a green region at $\mathrm{C} 7$ indicating the requirement for bulky substituents at this region for enhancing potency of the 2 inhibitor. Bulky substitution is essential when green polygon exists and prohibited when it is yellow [57]. The qualitative SAR for compounds 2-6 indicates that the introduction of cyclopentane ring improves the inhibition efficiency compared to cyclobutane, Table 1 . However, the sterically disfavored yellow contours for compounds 3, 4, 6 at C16, 17, 19 atoms indicate reduction of the activity upon substitution with bulky groups at these positions. As depicted in Figure 6, for compound 2, the green polygon on the side of the phenol ring will interact with the hydrophobic residue (Leu419, Met423) which increase binding between the inhibitor and receptor. An inspection of Figs. 5-7 indicates that substitution with electron rich groups is expected to improve the inhibitory effect as shown by the red color in Figure 6 for compounds 3,6=>50,52 $\mu \mathrm{M}$ compared to $2,4=>100,93 \mu \mathrm{M}$, Table 1$)$, hydrophobicity increased in Figs. 5,7. This might interpret the increase in the potency when adding aromatic or hetero atoms such as sulfur atom, Table 1. For CoMSIA field, any hydrophobic, acceptor fields are included in the steric field. However, the donor field is embedded in the electrostatic field as concluded from the tripos bookshelf for sybyl (sybyl's manual). The hydrophobic disfavored (gray) over methyl C7 for 2; C17 (gray) disfavored hydrophobic, C4 (orange) favored hydrophobic, C7, C16 (yellow) disfavored steric for inhibitor 3; yellow, gray contours over cyclobutane for compound 4 and finally (yellow) over part of cyclohexane, (orange) favored hydrophobic on C15 for inhibitor 6 . These findings may explain comparable activity of compounds 2, 4: of 100, $93 \mu \mathrm{M}$ and compounds 3, 6: 50, 52 $\mu \mathrm{M}$ as displayed in Figure 4 and Table 1. 


\section{pharmacophore features and IC 50 per each compound}

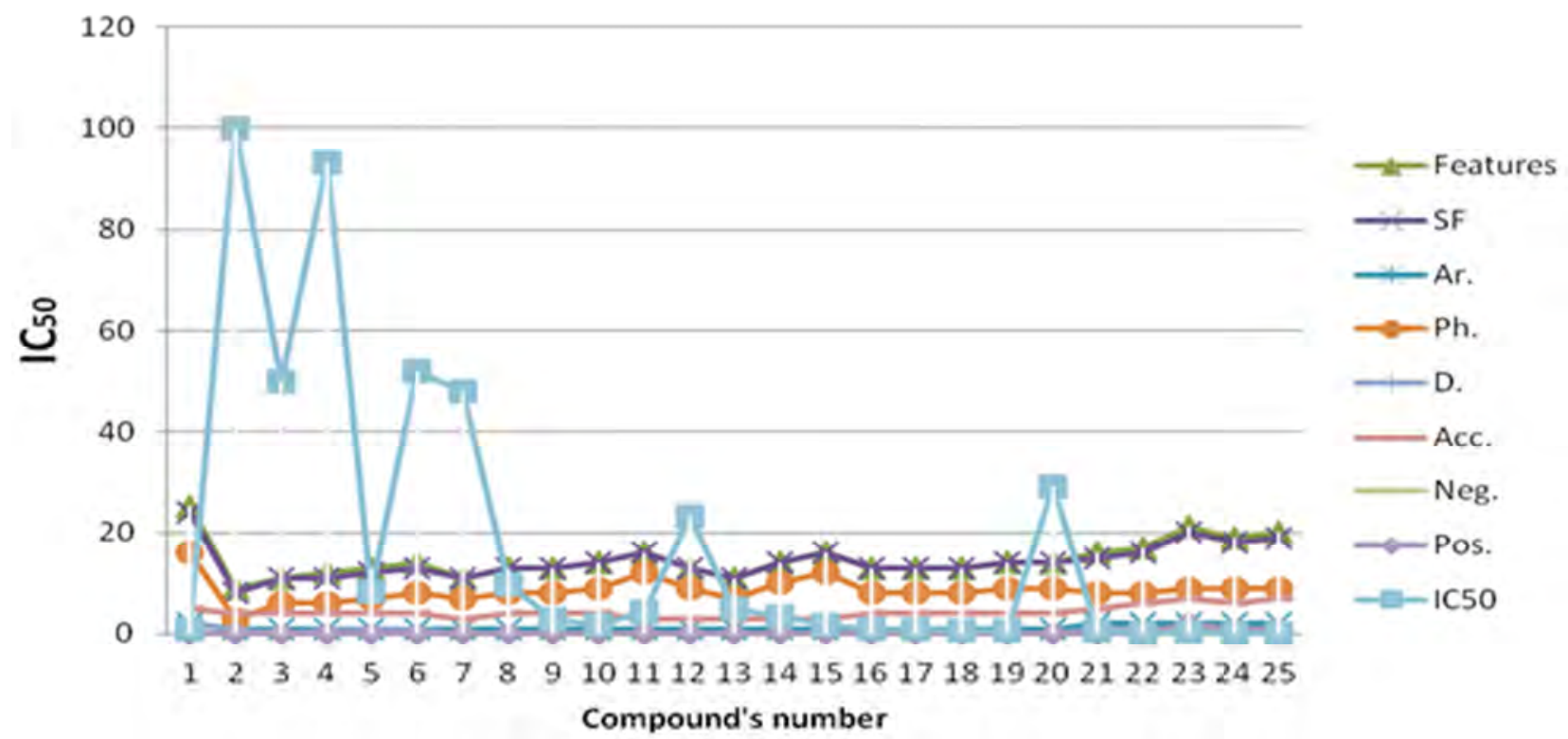

Figure 6. Pharmacophore mapping of 25 structures for Thumb pocket-II including spatial features (SF) aromatic centers (Ar.), hydrophobic centers (ph), Donor atoms (D.), Acceptor (Acc.), Negative (Neg.), Positive (Pos) and IC $C_{50}(\mu M)$.

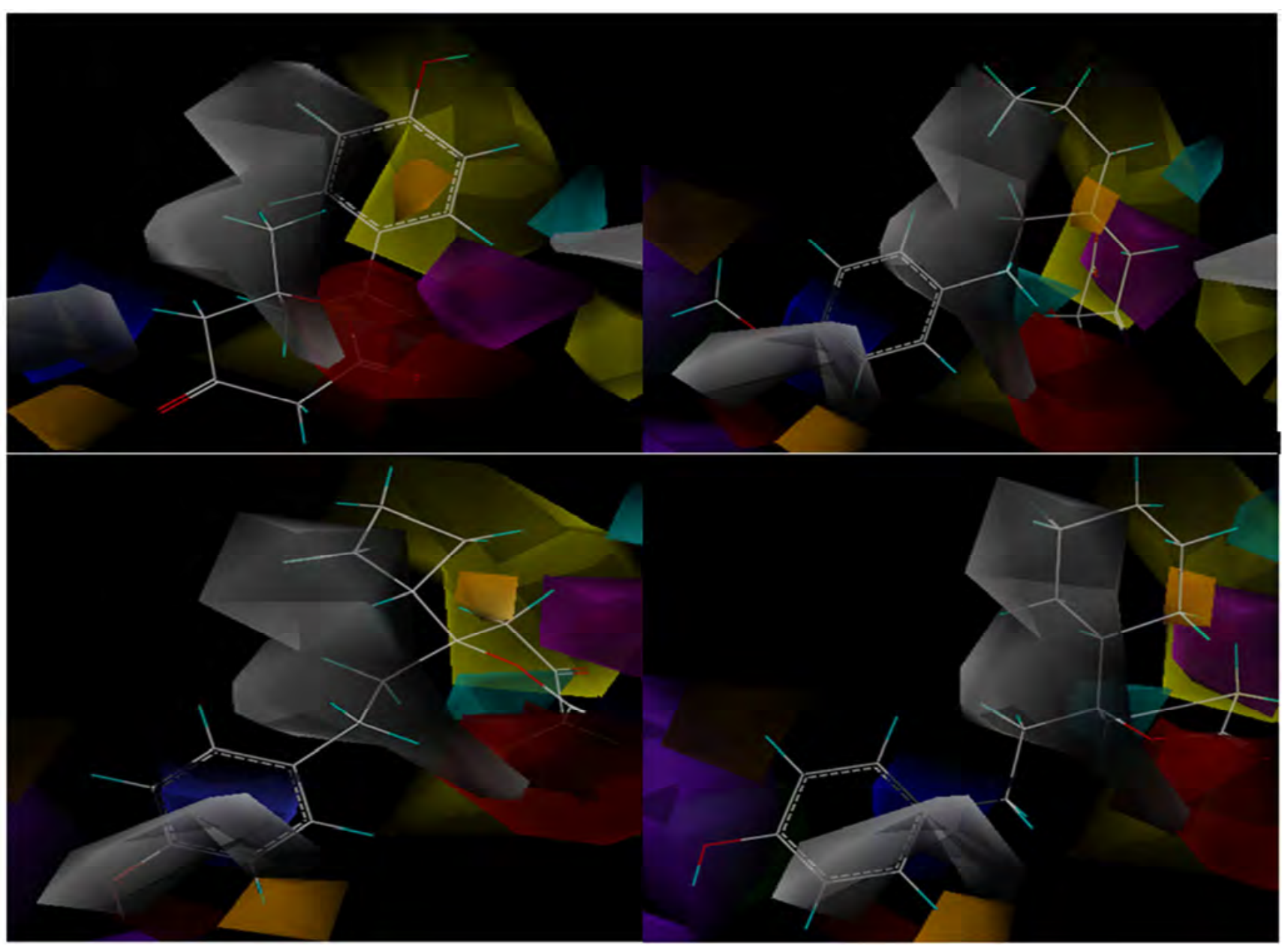

Figure 7. Contour maps around compound 2,3,4 and 6. 
Table 2. Partial least square (PLS) analysis of CoMFA and CoMSIA models

\begin{tabular}{|c|c|c|c|c|c|c|c|}
\hline \multirow{2}{*}{ PLS analysis $\backslash$ model } & \multirow{2}{*}{$\begin{array}{l}\text { CoMFA } \\
\text { SE }\end{array}$} & \multicolumn{6}{|c|}{ CoMSIA } \\
\hline & & SE & SEH & SEDA & SEDAH & DA & DAH \\
\hline$\overline{Q^{2}}$ & 0.43 & 0.34 & 0.36 & 0.51 & 0.52 & 0.46 & 0.65 \\
\hline Component & 5 & 6 & 10 & 6 & 7 & 6 & 10 \\
\hline $\mathrm{R}^{2}$ & 0.98 & 0.967 & 0.99 & 0.96 & 0.99 & 0.79 & 0.98 \\
\hline SEE & 0.162 & 0.201 & 0.061 & 0.275 & 0.108 & 0.508 & 0.175 \\
\hline F value & 162.59 & 87.14 & 587.63 & 74.28 & 187.02 & 11.11 & 69.84 \\
\hline Steric & 0.428 & 0.369 & 0.171 & 0.152 & 0.099 & - & - \\
\hline HB Donor & - & - & - & 0.363 & 0.254 & 0.586 & 0.231 \\
\hline HB Acceptor & - & - & - & 0.179 & 0.143 & 0.414 & 0.499 \\
\hline Hydrophobic & - & - & 0.402 & - & 0.244 & - & - \\
\hline
\end{tabular}

$\mathrm{HB}=$ hydrogen bond, $\mathrm{S}=$ steric, $\mathrm{E}=$ electrostatic, $\mathrm{D}=$ hydrogen bond donor $\mathrm{A}=$ hydrogen bond acceptor, $\mathrm{H}=$ hydrophobic

Table 3. Actual and predicted IC $_{50}$ from CoMFA and CoMSIA models.

\begin{tabular}{|c|c|c|c|c|c|}
\hline Inhibitor & $\mathrm{pIC}_{50}$ & CoMFA_SE & Residual & CoMSIA_SEADH & Residual \\
\hline 1 & 6.0315 & 5.921 & 0.1105 & 6.03 & 0.0015 \\
\hline 2 & 4 & 4.1131 & -0.1131 & 4.0193 & -0.0193 \\
\hline 3 & 4.301 & 4.2963 & 0.0047 & 4.295 & 0.006 \\
\hline 4 & 4.0315 & 4.316 & -0.2845 & 4.0221 & 0.0094 \\
\hline 5 & 5.0862 & 4.9775 & 0.1087 & 5.1017 & -0.0155 \\
\hline 6 & 4.284 & 4.2218 & 0.0622 & 4.2309 & 0.0531 \\
\hline 7 & 4.3188 & 4.4509 & -0.1321 & 4.2553 & 0.0635 \\
\hline 8 & 5.0132 & 5.2743 & -0.2611 & 5.1236 & -0.1104 \\
\hline 9 & 5.5376 & 5.3977 & 0.1399 & 5.5936 & -0.056 \\
\hline 10 & 5.7696 & 5.8175 & -0.0479 & 5.7071 & 0.0625 \\
\hline 11 & 5.3665 & 5.4548 & -0.0883 & 5.4426 & -0.0761 \\
\hline 12 & 4.6383 & 4.6319 & 0.0064 & 4.8441 & -0.2058 \\
\hline 13 & 5.301 & 4.9552 & 0.3458 & 5.2831 & 0.0179 \\
\hline 14 & 5.4437 & 5.4112 & 0.0325 & 5.3797 & 0.064 \\
\hline 15 & 5.7447 & 5.6632 & 0.0815 & 5.7965 & -0.0518 \\
\hline 16 & 6 & 5.8859 & 0.1141 & 6.0098 & -0.0098 \\
\hline 17 & 6.0506 & 5.8758 & 0.1748 & 5.7948 & 0.2558 \\
\hline 18 & 6.1024 & 6.1677 & -0.0653 & 6.1547 & -0.0523 \\
\hline 19 & 6.2757 & 6.4236 & -0.1479 & 6.2887 & -0.013 \\
\hline 20 & 4.5376 & 4.4839 & 0.0537 & 4.4972 & 0.0404 \\
\hline 21 & 6.2518 & 6.1485 & 0.1033 & 6.2336 & 0.0182 \\
\hline 22 & 6.8861 & 7.1074 & -0.2213 & 6.9216 & -0.0355 \\
\hline 23 & 6.5528 & 6.5735 & -0.0207 & 6.4622 & 0.0906 \\
\hline 24 & 6.8539 & 6.7979 & 0.056 & 6.8669 & -0.013 \\
\hline 25 & 7.4202 & 7.4323 & -0.0121 & 7.4446 & -0.0244 \\
\hline
\end{tabular}

Figure 7 shows Steric, electrostatic, hydrophobic, acceptor and donor contour maps around compound 2, 3, 4 and 6, respectively. Sterically favored areas are given in green, sterically unfavored areas in yellow; positive-charge-favored areas in blue, positive-charge-unfavored areas in red. Hydrophobic favored areas given in orange, hydrophobic unfavored areas in gray, donor-favored areas in cyan, donorunfavored areas in purple, acceptor-favored areas in magenta, acceptor unfavored areas in deep red. The maps generated depict regions having scaled coefficients $>80 \%$ (favored) or $<20 \%$ (disfavored).

\section{Conclusions}

The present work provides an analysis of the interaction between $\mathrm{HCV} \mathrm{NS} 5 \mathrm{~b}$ and 25 compounds for better understanding of the reported potency of these compounds. Molecular docking and 3D-QSAR models were used to study binding of the selected inhibitors with HCVNS5B allosteric sites. Hydrophobic interactions were found to be the most prominent factor for the interaction between the current inhibitors and HCV NS5B. This finding agrees with experiment. Substitutions at particular positions were explored based on electrostatic, steric, and hydrophobic interactions.

\section{References}

[1] P. Simmonds, J. Mellor, T. Sakuldamrongpanich, C. Nuchaprayoon, S. Tanprasert, E. C. Holmes, D. B. Smith, Evolutionary analysis of variants of hepatitis $\mathrm{C}$ virus found in South-East Asia: comparison with classifications based upon sequence similarity, J. Gen. Virol., 77 (1996) 3013-3024.

[2] S. Ramia, J. Eid-Fares, Distribution of hepatitis C virus genotypes in the Middle East Int. J. Infect. Dis., 10 (2006) 272-277.

[3] J. P. Watson, H. Al-Mardini, S. Awadh, S. Ukabam, C. O. Record, Hepatitis C virus genotypes in a cohort of Middle Eastern patients, Ann. Saudi. Med., 19 (1999) 410-412. 
[4] F. Fallahian, A. Najafi, Epidemiology of Hepatitis C in the Middle East, Saudi J. Kidney Dis. Transpl., 22 (2011) 1-9.

[5] A. Craxì, J. Pawlotsky, H. Wedemeyer, K. Bjoro, R. Flisiak, X. Forns, M. Mondelli, M. Peck-Radosavljevic, W. Rosenberg, C. Sarrazin, EASL Clinical Practice Guidelines: Management of hepatitis C virus infection, J. Hepatol., 55 (2011) 245-264.

[6] M. Yahia, Global health: a uniquely Egyptian epidemic, Nature, 474 (2011) S12-S13.

[7] F. El-Zanaty, W. Ann, Egypt Demographic and Health Survey 2008 Cairo, Egypt: Ministry of Health, El-Zanaty Associates, and Macro International, (2009).

[8] B. R. Mauss, W. Sarrazin, Short Guide to Hepatitis C, the Flying Publisher, Bernd Kamps Steinhäuser Verlag, 2011, pp. 128.

[9] M. A. Kamel, Y. A. Ghaffar, M. A. Wasef, M. Wright, L. C. Clark, F. D. Miller, High HCV prevalence in Egyptian Blood donors, Lancet., 340 (1992) 427.

[10] C. Frank, M. K. Mohamed, G. T. Strickland, D. Lavanchy, R. R. Arthur, L. S. Magder, T. El Khoby, Y. Abdel-Wahab, E. Aly Ohn, W. Anwar, I. Sallam, The role of parenteral antischistosomal therapy in the spread of hepatitis $\mathrm{C}$ virus in Egypt, Lancet 355 (2000) 887-891.

[11] M. Karmochkine, F. Carrat, O. Dos Santos, P. Cacoub, G. Raguin, A case-control study of risk factors for hepatitis C infection in patients with unexplained routes of infection, $\mathrm{J}$. Viral. Hepat., 13 (2006) 775-782.

[12] M. K. Mohamed, M. Abdel-Hamid, N. N. Mikhail, F. AbdelAziz, A. Medhat, L. S. Magder, A. D. Fix, G. T. Strickland, Intrafamilial transmission of hepatitis C in Egypt, Hepatol., 42 (2000) 683-687.

[13] M. J. Alter, Epidemiology of hepatitis C virus infection, World J. Gastroenterol, 13 (2007) 2436-2441.

[14] T. A. Steitz, DNA Polymerases: Structural Diversity and Common Mechanisms, J. Biol. Chem., 274 (1999) 1739517398.

[15] D. Das, J. Hong, S. Chen, G. Wang, L. Beigelman, S. D. Seiwert, B. O. Buckman, Recent advances in drug discovery of benzothiadiazine and related analogs as $\mathrm{HCV}$ NS5B polymerase inhibitors, Bioorg. Med. Chem., 19 (2011) 46904703.

[16] L. Tomei, S. Altamura, G. Paonessa, R. De Francesco, G. Migliaccio, HCV antiviral resistance: the impact of in vitro studies on the development of antiviral agents targeting the viral NS5B polymerase, Antivir. Chem. Chemother., 16 (2005) 225-245.

[17] S. Betzi, C. Eydouxc, C. Bussetta, M. Blemont, P. Leyssen, C. Debarnot, M. Ben-Rahou, J. Haiech, M. Hibert, F. Gueritte, D. S. Grierson, J.-L. Romette, J.-C. Guillemot, J. Neyts, K. Alvarez, X. Morelli, H. Dutartre, B. Canard, Identification of allosteric inhibitors blocking the hepatitis $\mathrm{C}$ virus polymerase NS5B in the RNA synthesis initiation step, Antiviral Res., 84 (2009) 48-59.

[18] H. Li, J. Tatlock, A. Linton, J. Gonzalez, A. Borchardt, P. Dragovich, T. Jewell, T. Prins, R. Zhou, J. Blazel, H. Parge, R. Love, M. Hickey, C. Doan, S. Shi, R. Duggal, C. Lewis, S. Fuhrman, Identification and structure-based optimization of novel dihydropyrones as potent $\mathrm{HCV}$ RNA polymerase inhibitors, Bioorg. Med. Chem. Lett., 16 (2006) 4834-4838.

[19] R. De Francesco, A. Carfí, Advances in the development of new therapeutic agents targeting the NS3-4A serine protease or the NS5B RNA-dependent RNA polymerase of the hepatitis C virus, Adv. Drug. Deliv. Rev., 59 (2007) 1242 1262.

[20] C. Vasquez, Allosteric effects of the GTP-specific binding site and a benzimidazole-derivative nonnucleoside inhibitor on the hepatitis C virus RNA-dependent RNA polymerase, Microbiology and Immunology, McGill University, Montreal, 2009.

[21] U. Koch, F. Narjes, Allosteric Inhibition of the Hepatitis C Virus NS5B RNA Dependent RNA Polymerase, Infect. Disord. Drug. Targets., 6 (2006) 31-41.

[22] S. Colarusso, B. Attenni, B. Attenni, S. Avolio, S. Malancona, S. Harper, S. Altamura, U. Koch, F. Narjes, Inhibitors of the hepatitis C virus RNA-dependent RNA polymerase, ARKIVOC vii (2006) 479-495.

[23] M. H. Powdrill, J. A. Bernatchez, M. Götte, Inhibitors of the Hepatitis C Virus RNA-Dependent RNA Polymerase NS5B, Viruses 2(2010) 2169-2195.

[24] J. Vermehren, C. Sarrazin, New HCV therapies on the horizon, Clin. Microbiol. Infect., 17 (2011) 122-134. D. B. Kitchen, H. Decornez, J. R. Furr, J. Bajorath, Docking and scoring in virtual screening for drug discovery: methods and applications, Nature reviews. Drug discovery, 3 (2004) 935-949.

[25] C. Levinthal, S. Wodak, P. Kahn, A. Dadivanian, Hemoglobin interaction in sickle cell fibers. I: Theoretical approaches to the molecular contacts, Proc Natl Acad Sci 72 (1975) 13301334.

[26] K. H. Kim, G. Greco, E. Novellino, A Critical Review of Recent CoMFA Applications 3D QSAR in Drug Design, in: H. Kubinyi, G. Folkers, Y. C. Martin (Eds.), Springer Netherlands2002, pp. 257-315.

[27] H. Kubinyi, QSAR and 3D QSAR in drug design Part 1: methodology, Drug Discovery Today, 2 (1997) 457-467.

[28] L. Zhang, K. C. Tsai, L. Du, H. Fang, M. Li, W. Xu, How to generate reliable and predictive CoMFA models, Curr. Med. Chem., 18 (2011) 923-930.

[29] J. C. Shelley, A. Cholleti, L. Frye, J. R. Greenwood, M. R. Timlin, M. Uchimaya, Epik: a software program for pKa prediction and protonation state generation for drug-like molecules, J. Comp.-Aided Mol. Design, 21 (2007) 681-691.

[30] A. E. Cho, D. Rinaldo, Extension of QM/MM docking and its applications to metalloproteins, J. Comput. Chem., 30 (2009) 2609-2616.

[31] R. Farid, M. Ringnalda, M. Murcko, D. J. Weiser, Y. Tran, M. Byington, W. A. Glauser, S.-Y. Liu, A. Monge, S. Ozawa, L. Frye, W. Sherman, V. Eyrich, Y. Shimada, S. Becker, R. Abel, J. Vertrees, QM-Polarized Ligand Docking : A novel research solution that combines the power of Glide with the accuracy of QSite, in: R. Friesner, P. Anderson, B. J. Berne, W. C. Guida, B. Honig, M. P. Jacobson, W. L. Jorgensen, R. M. Levy (Eds.) Schrödinger is the scientific leader in computational chemistry, providing software solutions and services for life sciences and materials research, 2012. 
[32] R. Thomsen, M. H. Christensen, MolDock: A New Technique for High-Accuracy Molecular Docking, J. Med. Chem., 49 (2006) 3315-3321.

[33] A. E. Cho, V. Guallar, B. Berne, R. A. Friesner, Importance of Accurate Charges in Molecular Docking: Quantum Mechanical/Molecular Mechanical (QM/MM) Approach, J. Comput. Chem., 26 (2005) 915-931.

[34] A.-S. S. H. Elgazwy, N. S. M. Ismail, H. S. A. Elzahabi, 45 A convenient synthesis and molecular modeling study of novel purine and pyrimidine derivatives as CDK2/cyclin A3 inhibitors, Bioorg Med Chem, 18 (2010) 7639-7650.

[35] M. ElHefnawi1, M. ElGamacy, M. Fares, Multiple virtual screening approaches for finding new Hepatitis c virus RNAdependent RNA polymerase inhibitors: Structure-based screens and molecular dynamics for the pursue of new poly pharmacological inhibitors, BMC Bioinformatics 13 (2012) S5.

[36] M. S. Bahia, S. K. Gunda, S. R. Gade, S. Mahmood, R. Muttineni, O. Silakari, Anthranilate derivatives as TACE inhibitors: docking based CoMFA and CoMSIA analyses, J Mol Model, 17 (2011) 9-19.

[37] S. Boppana, N. S. Pagadala, K. S. Rangappa, structure based designing of new inhibitors a gainst acetylcholine esterase associated with Alzheimer's disease, in: K. S. Rangappa (Ed.) structure based designing of new inhibitors, 2009, pp. 29-36.

[38] G. A. Balaji1, V. N. Balaji, S. N. Rao, Utility of scoring function customization in docking-based virtual screening approaches, Curr. Sci., 104 (2013).

[39] V. Sobolev, A. Sorokine, J. Prilusky, E. E. Abola, M. Edelman, Automated analysis of interatomic contacts in proteins, Bioinformatics, 15 (1999) 327-332.

[40] E. F. Pettersen, T. Goddard, C. Huang, G. S. Couch, D. M. Greenblatt, E. C. Meng, T. Ferrin, E., UCSF Chimera: a visualization system for exploratory research and analysis, $\mathrm{J}$. Comput. Chem., 25 (2004) 1605-1612.

[41] H. Abedi, H. Ebrahimzadeh, J. B. Ghasemi, 3D-QSAR, CoMFA, and CoMSIA of new phenyloxazolidinones derivatives as potent HIV-1 protease inhibitors, Structural Chemistry, 24 (2013) 433-444.

[42] J. Gasteiger, M. Marsili, Iterative partial equalization of orbital electronegativity - a rapid access to atomic charges, Tetrahedron, 36 (1980) 3219-3228.

[43] J. L. Medina-Franco, S. Rodríguez-Morales, C. JuárezGordiano, A. Hernández-Campos, R. Castillo, Docking-based CoMFA and CoMSIA studies of non-nucleoside reverse transcriptase inhibitors of the pyridinone derivative type, Journal of Computer-Aided Molecular Design, 18 (2004) 345360 .

[44] C. Pargellis, L. Tong, L. Churchill, P. F. Cirillo, T. Gilmore, A. G. Graham, P. M. Grob, E. R. Hickey, N. Moss, S. Pav, J. Regan, Inhibition of p38 MAP kinase by utilizing a novel allosteric binding site, Nat Struct Biol, 9 (2002) 268-272.

[45] V. N. Viswanadhan, A. K. Ghose, G. R. Revankar, R. K. Robins, Atomic physicochemical parameters for three dimensional structure directed quantitative structure-activity relationships. 4. Additional parameters for hydrophobic and dispersive interactions and their application for an automated superposition of certain naturally occurring nucleoside antibiotics, Journal of Chemical Information and Computer Sciences, 29 (1989) 163-172.

[46] S. Bressanelli, L. Tomei, F. A. Rey, R. De Francesco, Structural analysis of the hepatitis $\mathrm{C}$ virus RNA polymerase in complex with ribonucleotides, J. Virol., 76 (2002) 3482-3492.

[47] A. E. Gorbalenya, F. M. Pringle, J. L. Zeddam, B. T. Luke, C. E. Cameron, J. Kalmakoff, T. N. Hanzlik, K. H. Gordon, V. K. Ward, The palm subdomain-based active site is internally permuted in viral RNA-dependent RNA polymerases of an ancient lineage, J. Mol. Biol., 324 (2002) 47-62.

[48] E. K. O'Reilly, C. C. Kao, Analysis of RNA-dependent RNA polymerase structure and function as guided by known polymerase structures and computer predictions of secondary structure, Virology 252 (1998) 287-303.

[49] C. A. Lesburg, R. Radfar, P. C. Weber, Recent advances in the analysis of HCV NS5B RNA-dependent RNA polymerase, Curr. Opin. Investig. Drugs., 1 (2000) 289-296.

[50] C. A. Brautigam, T. A. Steitz, Structural and functional insights provided by crystal structures of DNA polymerases and their substrate complexes, Curr. Opin. Struct. Biol., 8 (1998) 54-63.

[51] N. Ai-hua, Recent advances in HCV NS5B RNA-dependent RNA polymerase inhibitors, Journal of International Pharmaceutical Research, 39 (2012) 89-103.

[52] T. Li, M. Froeyen, P. Herdewijn, Insight into ligand selectivity in HCV NS5B polymerase: molecular dynamics simulations, free energy decomposition and docking, J. Mol. Model., 16 (2010) 49-59.

[53] D. Plewczynski, M. Łaźniewski, R. Augustyniak, K. Ginalski, Can we trust docking results? Evaluation of seven commonly used programs on PDBbind database, J. Comput. Chem., 32 (2011) 742-755.

[54] R. A. Love, H. E. Parge, X. Yu, M. J. Hickey, W. Diehl, J. Gao, H. Wriggers, A. Ekker, L. Wang, J. A. Thomson, P. S. Dragovich, S. A. Fuhrman, Crystallographic identification of a noncompetitive inhibitor binding site on the hepatitis $\mathrm{C}$ virus NS5B RNA polymerase enzyme, J. Virol., 77 (2003) 75757581 .

[55] A. Arleo, A. Mangia, The current treatment of Hepatitis C, Minerva gastroenterologica e dietologica, 62 (2016) 167-182.

[56] C. J. Monceaux, C. Hirata-Fukae, P. C. Lam, M. M. Totrov, Y. Matsuoka, P. R. Carlier, Triazole-linked reduced amide isosteres: an approach for the fragment-based drug discovery of anti-Alzheimer's BACE1 inhibitors, Bioorganic \& medicinal chemistry letters, 21 (2011) 3992-3996.

[57] S. Duhovny, D. Dror, O. Inbar, Y. Nussinov, R. Wolfson, J. Haim, PharmaGist: a webserver for ligand-based pharmacophore detection, Nucleic Acids Res., 36 (2008) W223-W228.

[58] S. V. Damme, Quantum chemistry in QSAR : Quantum chemical descriptors :Use, benefits and drawbacks, Department of Inorganic and Physical Chemistry, Ghent University, Ghent, 2009, pp. 349. 